\title{
Catalogue of Cassidinae (Coleoptera: Chrysomelidae) from the New Leaf Beetles Collection from "Grigore Antipa" National Museum of Natural History (Bucharest) (Part II)
}

\author{
Sanda MAICAN ${ }^{1, *}$, Rodica SERAFIM ${ }^{2}$ \\ ${ }^{1}$ Institute of Biology Bucharest of Romanian Academy, 296 Splaiul Independenţei, 060031 Bucharest, \\ P.O. Box 56-53, Romania. \\ 2"Grigore Antipa" National Museum of Natural History, Kiseleff 1, 011341 Bucharest, Romania. \\ *corresponding author, e-mail: sanda.maican@ibiol.ro
}

Received: June 30, 2017; Accepted: November 7, 2017; Available online: December 28, 2017; Printed: December 31, 2017

\begin{abstract}
The paper reports data on the species of Cassidinae preserved in the new Chrysomelidae Collection of "Grigore Antipa" National Museum of Natural History (Bucharest). A total of 852 specimens, representing four genera and 31 species are listed. Out of them, 11 species are recently entered in this collection. New distributional data for some rare species of Cassida in Romanian fauna are given.
\end{abstract}

Key words: Chrysomelidae, Cassidinae, collections, "Grigore Antipa" National Museum of Natural History, Bucharest.

\section{INTRODUCTION}

The subfamily Cassidinae includes about $16 \%$ of leaf beetle species diversity and forms the second largest clade of Chrysomelidae family, after Galerucinae (Chabo, 2007).

In old sense, Cassidinae (commonly named tortoise beetles) and Hispinae (leaf-mining beetles) were considered distinct subfamilies (Seeno \& Wilcox, 1982). Cassidinae s.str. includes 2,906 species from 154 genera placed in 19 tribes (Seeno \& Wilcox, 1982) or 12 tribes (Borowiec, 1999), and Hispinae s.str. comprises 2,980 species grouped in 170 genera and 24 tribes (Seeno \& Wilcox, 1982; Staines, 2002; Chabo, 2007).

The combination of Cassidinae with Hispinae is relatively recent (Staines, 2002). Thus, the subfamily Cassidinae sensu lato includes 6,319 described species (3,371 hispines and 2,948 cassidines) distributed worldwide. According to Borowiec \& Świętojańska (2014), Cassidines are classified in 341 genera and 36 tribes.

A total of 32 species of Cassidinae from four genera (Cassida Linnaeus, Hypocassida Weise, Pilemostoma Desbrochers des Loges and Hispa Linnaeus) were reported in Romanian fauna (Maican, 2005; Sekerka, 2007).

In "Grigore Antipa" National Museum of Natural History, the Cassidinae material is included in the Collection of Palaearctic Coleoptera (data have been published partially) and in the recently formed Collection of Chrysomelidae. The study and arrangement of the material in the new leaf-beetle collection started in 2016. Thus, in the first part of the catalogue of this collection, data on 33 Palaearctic species of subfamilies Donaciinae (tribes Donaciini, Haemoniini and Plateumarini) and Criocerinae were published (Maican \& Serafim, 2016). 


\section{MATERIAL AND METHODS}

The new Cassidinae collection, which we refer in this paper, comprises:

- material that was not included in the Collection of Palaearctic Coleoptera, containing specimens from collections of Dénes Kenderessy (1846-1881), Edmund Reitter (1845-1920), Arnold Lucien Montandon (1852-1922), Friedrich Deubel (1845-1933), Richard Canisius (1872-1934), and Emil Várady. The Collection of Palaearctic Coleoptera was created around 1960, by gathering all the collections entered into the scientific heritage of "Grigore Antipa" Museum, at the end of the $19^{\text {th }}$ century and the beginning of the $20^{\text {th }}$ century;

- material collected from Romania (1951-2017) by the team of specialists from "Grigore Antipa" Museum and their collaborators during several research projects on the Romanian fauna;

- material from Dr. Nicolae Săvulescu (1920-1992) collection, which was included in the Museum patrimony after his death;

- specimens donated by Franz Salay (1861-1946), Igor Ceianu (1925-2000), Vladimir Brădescu (1915-2004), Mihai Şerban Procheş, Adrian Ruicănescu, Viorel Ungureanu, Sanda Maican;

- material collected during the scientific expeditions organized by "Grigore Antipa" Museum in the areas of the Mediterranean Basin: "Punia" 2006 (Tunisia) and "Euphrates" 2008 (Turkey).

The paper presents the results of the study of the cassidines material (tribes Cassidini and Hispini) preserved in the new Chrysomelidae collection of the "Grigore Antipa" National Museum of Natural History, Bucharest.

Parts of the material of Chrysomelidae were published by Negru \& Roşca (1967), Negru (1968), Hoinic (1994), Maican \& Serafim (2001, 2004, 2009, 2012), Maican (2006, 2007 a, b), Serafim \& Maican (2004, 2008, 2011), Ungureanu et al. (2008).

The species identification was made by Atena Roșca ${ }^{1}$, Igor Ceianu ${ }^{2}$, Cristina Hoinic $^{3}$, Sanda Maican, Rodica Serafim, Ștefan Negru ${ }^{4}$ and Mircea Alexandru Ieniștea ${ }^{5}$. For identification, the following papers were consulted: Warchałowski $(2003,2010)$, Sekerka (2010), Borowiec (2013), Borowiec \& Świętojańska (2002-2017).

Nomenclature and systematical order follows the Catalogue of Palaearctic Coleoptera (Borowiec \& Sekerka, 2010).

The stereomicroscope Stemi 2000-C was used to identify the specimens, and habitus photos were taken using the Canon EOS 5D Mark IV digital camera.

For each species, the collecting site, date, the number of the specimens, the collectors' name and the host plants are mentioned.

Abbreviations:

coll. - collection; f.c. - Forest chalet; Mt. (Mts) - Mountain/Mountains; m a.s.1. - meters above sea level; spec. (s) - specimen/specimens.

Names of the collectors:

1'Atena Roşca (1930-1976), worked at the Museum between 1954 and 1976.

${ }^{2}$ Igor Ceianu (1925-2000), a Romanian specialist of plant protection.

${ }^{3}$ Cristina Hoinic (1967-1997), worked at the museum between 1992 and 1997.

${ }^{4}$ Ştefan Negru (1923-1970), worked at the Museum between 1963 and 1970.

${ }^{5}$ Mircea Alexandru Ieniştea (1910-1994), a renowned entomologist and professor, collaborator of the Museum. 
A.C. - Alin Constantinescu; A.L. - Alexandra Levardă (Popa); Ad.R. - Adrian Ruicănescu; A.M.K. - Ana Maria Krapal; A.P. - Angela Petrescu; A.Pe. - Angela Petrişor; A.G. - Aristiţa Goagă; A.L.M. - Arnold Lucien Montandon; A.R. - Atena Roşca; A.P.G. - Aurelian Popescu - Gorj; A.S. Aurora Stănescu; A.Z. - A. Zoppa; C.P. - Corneliu Pârvu; C.B. - Cristina Ban (Calefariu); C.C. - Corina Clisu; C.H. - Cristina Hoinic; D.D. - Dan Dumitrescu; D.K. - Dénes Kenderessy; D.M. - Dumitru Murariu; D.S. - Dragoș Scăunaşu; Ed.R. - Edmund Reitter; E.I. - Elena Iorgu; E.R. - Elena Rusu; E.V. - Emil Váradi; F.S. - Franz Salay; Fr.D. - Friedrich Deubel; G.A. - Gabriela Andrei; G.C. - Gabriel Chişamera; I.A. - Ioana Andreescu; I.C. - Igor Ceianu; I.D. - Ion Drăghia; Io.C. - Iosif Căpuşe; I.M. - Ioana Matache; I.N. - Ioan Nemeş; I.S. - Igor Sienkiewicz; I.Şt.I. - Ionuţ Ştefan Iorgu; L.O.P. - Luis Ovidiu Popa; M.A. - Mircea Andrei; M.I. - Maria Iacob; M.V. - Marin Voicu; M.B. - Mihaela Barcan (Achim); M.W. - Medeea Weinberg; M.S. - Melanya Stan; M.S.P. - Mihai Şerban Procheş; N.R. Năstase Răduleț; N.G. - Nicolae Găldean; N.S. - Nicolae Săvulescu; P.G. - Pante Gherghel; R.A. - R. Albrecht; R.C. - Richard Canisius; R.S. - Rodica Serafim; S.St. - Steluţa State; Şt.N. - Ştefan Negru; Th.W. - Th. Wanka; V.U. - Viorel Ungureanu; V.B. - Vladimir Brădescu; V.G. - Victor Gheorghiu; X.S.P. - Xenia Scobiola Palade.

Abbreviations of the county names in Romania:

AB - Alba; AG - Argeş; BC - Bacău; BR - Brăila; BV - Braşov; BZ - Buzău; CS - Caraş Severin; CL - Călăraşi; CJ - Cluj; CT - Constanţa; DB - Dâmboviţa; DJ - Dolj; GL - Galaţi; GJ - Gorj; GR - Giurgiu; HD - Hunedoara; HR - Harghita; IF - Ilfov; IL - Ialomiţa; IS - Iaşi; MH - Mehedinţi; MM - Maramureş; MS - Mureş; NT - Neamţ; PH - Prahova; SB - Sibiu; SJ - Sălaj; SM - Satu Mare; SV - Suceava; TL - Tulcea; TR - Teleorman; VS - Vaslui; VL - Vâlcea; VN - Vrancea. Bucureşti Bucharest (capital city of Romania).

\section{RESULTS}

The Chrysomelidae Collection of the "Grigore Antipa" National Museum of Natural History (Bucharest) includes 852 specimens representing 31 species belonging to four genera of Cassidinae, presented below. The species newly entered in the Museum collections are marked with*.

Family Chrysomelidae Latreille, 1802

Subfamily Cassidinae Gyllenhal, 1813

Tribe Cassidini Gyllenhal, 1813

Cassida Linnaeus, 1758

Cassida atrata Fabricius, 1787

Material: 1 spec., Tuşnad (HR), without other data, coll. I.C. (Fig. 1).

Host plants: Lamiaceae: Salvia glutinosa, S. pratensis (Borowiec, 2013).

*Cassida aurora Weise, 1907

Material: $1 \mathrm{spec} .$, Maribor (Marburg), Slovenia, without other data, coll. Fr.D. (Fig. 2).

Host plants: Asteraceae: Achillea millefolium, A. ptarmica (Borowiec, 2013).

*Cassida azurea Fabricius, 1801

Material: 1 spec., Săbăreni (GR), 17.V.1958, I.S; 1 spec., Mezö Záh (Zau de Câmpie) (MS), Zoppa, coll. Fr.D.

Host plants: Caryophyllaceae: Saponaria officinalis, Silene alba, S. behen, $S$. conica, S. vulgaris (Borowiec, 2013).

*Cassida berolinensis Suffrian, 1844

Material: 1 spec., Jegălia (CL), 26.V.1955, coll. I.C. (Fig. 3).

Host plants: Caryophyllaceae: Minuartia viscosa; Chenopodiaceae: Atriplex sphaeromorpha, Chenopodium album, Salsola collina (Borowiec, 2013). 
Cassida canaliculata Laicharting, 1781

Material: 2 specs, Comana Forest (GR), without other data, coll. A.L.M.; 1 spec., Câmpulung Moldovenesc (SV), 13.IV.1953, coll. I.C.; 3 specs, Floristic Reserve "The Secular Hayfields from Valea lui David”, Iaşi (IS), 10.V.2012, I.Şt.I.; 2 specs, the Natural Reserve "Marine Sand Dunes of Agigea" (CT), 23.V.2012, I.Şt.I.; 1 spec., Haşag (SB), 26.V.2015, E.I.; 1 spec., Făcăeni (IF), 21.V.2017, G.C. (Fig. 4).

Host plants: Lamiaceae: Salvia pratensis, S. verticillata (Borowiec, 2013).

Cassida deflorata Suffrian, 1844

Material: 1 spec., Tunisia, near Bou Salem, Mejerda River, 27.III.2006, C.P. (Punia Expedition).

Host plants: Asteraceae: Arctium lappa, Carduus tenuiflorus, Cirsium dyris, Cynara cardunculus, C. scolymus, Senecio cineraria, Silbum marianum (Borowiec, 2013).

Cassida denticollis Suffrian, 1844

Material: 1 spec., Cehia, Moravia, Uherskỳ Brod (Ungarisch Brod), without other data, Th.W.; 1 spec., Cernica Forest, Bucureşti, 8.V.1962, I.D.; 1 spec., Chilia Veche (TL), 7.V.1964, M.W.; 1 spec., Cloşani (GJ), 16.VI.1992, C.P.; 1 spec., Canaraua Fetii (CT), 19.V.1993, C.H.; 1 spec., Dumbrăveni Forest (CT), 18.V.1994, C.H. (Fig. 5).

Host plants: Asteraceae: Achillea millefolium, Artemisia absinthium, A. campestris, Tanacetum corymbosum, T. vulgare (Borowiec, 2013).

Cassida ferruginea Goeze, 1777

Material: 1 spec., Cehia, Moravia, Uherskỳ Brod (Ungarisch Brod), without other data, Th.W.; 2 specs, Mirceşti (IS), 23.V.1968; 1 spec., Başta (NT), 23.V.1973. (Fig. 6).

Host plants: Asteraceae: Inula salicina, Pulicaria dysenterica, P. prostrata, P. uliginosa (Borowiec, 2013).

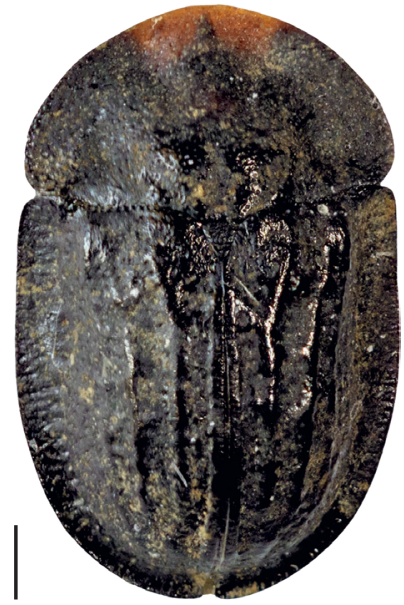

Fig. 1 - Cassida atrata (Photo I. Ș. Iorgu). Scale bar: $1 \mathrm{~mm}$.

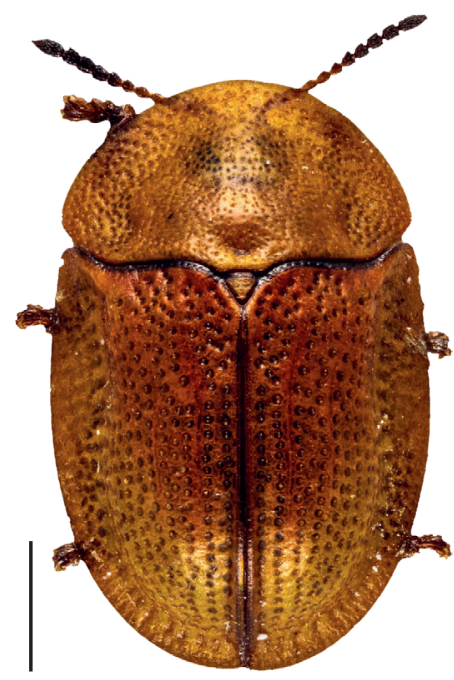

Fig. 2 - Cassida aurora (Photo I. Ș. Iorgu). Scale bar: $1 \mathrm{~mm}$. 
Cassida flaveola Thunberg, 1794

Material: 1 spec., without collecting data. In Romania, this species was mentioned from Haţeg, Sibiu, Arpad, Braşov (Petri, 1912), Zizin and Reghin (Kuthy, 1897). (Fig. 7).

Host plants: Caryophyllaceae: Cerastium vulgatum, Honckenya peploides, Malachium aquaticum, Spergula arvensis. S. nemorum, Stellaria graminea, S. media (Borowiec, 2013).

\section{Cassida hemisphaerica Herbst, 1799}

Material: 1 spec., Braşov, Tâmpa (Dealul Capelei = Kapellenberg) (BV), without other data, coll. Fr.D.; 1 spec., Bucovăț (DJ), 1.VIII.1951, X.S.P.; 1 spec., Sinaia (PH), IX.1957, I.S. (Fig. 8).

Host plants: Caryophyllaceae: Dianthus campestris, D. caryophyllus, Gypsophila paniculata, Saponaria officinalis, Silene cucubalus, S. flos-cuculi, S. latifolia, S. latifolia ssp. alba, S. natans, S. vulgaris (Borowiec, 2013).

\section{*Cassida lineola Creutzer, 1799}

Material: 1 spec., Hârşova (CT), without other data, coll. A.L.M.; 1 spec., without collecting data, coll. D.K.; 1 spec., Cerna (TL), 26.05.2005, C.B. (Fig. 9).

Host plants: Asteraceae: Artemisia absinthium, A. austriaca, A. campestris, A. scoparia (Borowiec, 2013.).

\section{*Cassida margaritacea Schaller, 1783}

Material: 2 specs, without collecting data, coll. D.K. In Romania, this species was recorded from Haşag, Sibiu and Tălmaciu (Kuthy, 1897). (Fig. 10).

Host plants: Caryophyllaceae: Saponaria officinalis, Silene inflata, S. latifolia, S. vulgaris, Spergula arvensis; Asteraceae: Antennaria margaritacea, Centaurea nemoralis, C. scabiosa, C. cyanus, C. stenolepis, Helichrysum arenarium (Borowiec, 2013).

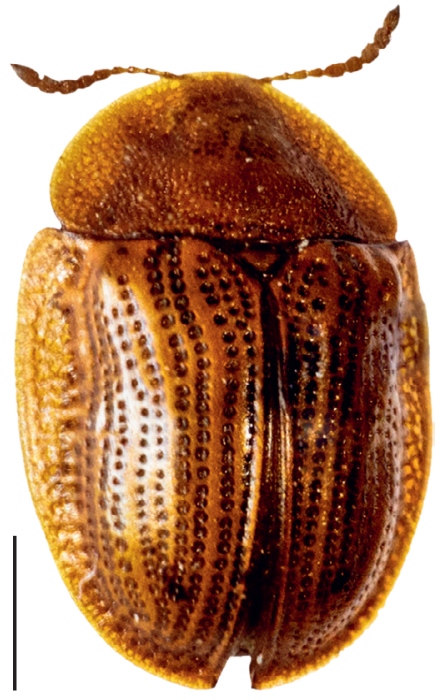

Fig. 3 - Cassida berolinensis (Photo I. Ș. Iorgu). Scale bar: $1 \mathrm{~mm}$.

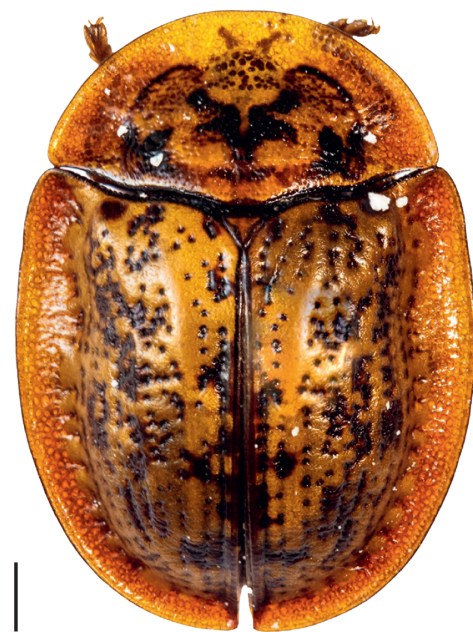

Fig. 4 - Cassida canaliculata (Photo I. Ș. Iorgu). Scale bar: $1 \mathrm{~mm}$. 
Cassida murraea Linnaeus, 1767

Material: 1 spec., Transylvania, without other data, coll. D.K.; 1 spec., Argeş (AG), without other data, coll. R.C.; 1 spec., Periş (IF), without other data, coll. R.C.; 6 specs, Comana Forest (GR), without other data, coll. A.L.M.; 1 spec., Brăila (BR), without other data, coll. A.L.M.; Cocioc, Bucureşti, 2.VII.1906, coll. F.S. (1 spec.), 25.VI.1907, coll. F.S. (1 spec.); Voineşti (DB), 24.V.1950, X.S.P. (1 spec.), 24.V.1954, X.S.P. (1 spec.); Pasărea Forest (IF), 4.V.1951, N.S. (1 spec.), 5.X.1966 (3 specs); 1 spec., Tulcea (TL), 3.VII.1952, N.S.; Herculane Baths (Băile Herculane) (CS), 5.VII.1953 (1 spec.), 18.V.1960, A.P.G. (3 specs), 19.V.1964, A.P.G. (2 specs), 17-25.V.1965, N.S., A.P.G. (6 specs), 28.V.1966, A.P.G. (1 spec.); 2 specs, Bârnova Forest (IS), 2 4.VI.1953, N.S.; Budeşti (CL), 28.VI.1954, X.S.P. (1 spec.), 9.VII.1957, N.S. (1 spec.); 1 spec., Băneasa Forest (IF), 4.V.1956, N.S.; 1 spec., Potelu (OT), 9.VI.1956; 1 spec., Cernica Forest (IF), 17.IV.1963, A.R.; 2 specs, Gâdinţi (NT), 11.V.1963-23.V.1965; 1 spec., Siseşti, Târgu Jiu (GJ), 7.07.1965; 1 spec., Stăniţa (NT), 7.V.1966; 1 spec., Ipoteşti (BT), 9.VI.1966, M.C.; Caraorman Forest, Danube Delta (TL), 9.V.1967-13. VI.1969, X.S.P. (2 specs), 24.VI.1991, P.G. (4 specs); Mirceşti (IS), 23.V.1968 (1 spec.), 1.VIII.1971 (1 spec.); 1 spec., Letea Forest, Danube Delta (TL), 12.VII.1971, N.S.; 1 spec., Eşelniţa (MH), 15.VI.1970, Şt.N.; Başta (NT), 17.V.1971 (1 spec.), 23.V.1973 (2 specs); 1 spec., Purani (TR), 28.IX.1979, G.A.; 1 spec., Tăuţii de Sus (MM), 12.IV.1985, X.S.P.; 2 specs, Poiana Neamţului (SB), 15.V.1986, I.M.; 1 spec., Călineşti Oaş (SM), 21.V.1987, R.S.; 1 spec., Știuculeasa Forest, Maxenu (BZ), 17.VII.1987, G.A.; 1 spec., Sihlea (VN), 23.VI.1987, C.P.; 2 specs, Maliuc, Danube Delta (TL), 25.VI.1991, Ad.R.; 1 spec., Cândești-Focşani (VN), 1.VIII.1994, Ad.R.; 1 spec., The hayfields of Cluj (Fânaţele Clujului) (CJ), 21.IV.1995, Ad.R.; Repedea f.c. (MM), 22.V.- 26.VI.1997, R.S., C.P., I.M. (4 specs); 1 spec., Elma clearing, Repedea (MM), 27.VI.1997, C.P.; 1 spec., Turkey, Zorkun Yaylas1 (Osmaniye), $1800 \mathrm{~m}$ a.s.1., 5.V.1998, M.S.P.; 2 specs, Pleşca f.c., Ocna Şugatag (MM), 17.VII.1998, A.Pe.; 1 spec., Poiana Lindini, Nera Gorges (CS), 15.V.2002, M.S.; Coşnea f.c., Poienile de sub Munte (MM), 16.VI.2003, C.P. (12 specs), R.S. (46 specs); 1 spec., Bentul Mic Cotoi Lake, Borduşani (IL), 22.VIII.2006, C.B. (Fig. 11).

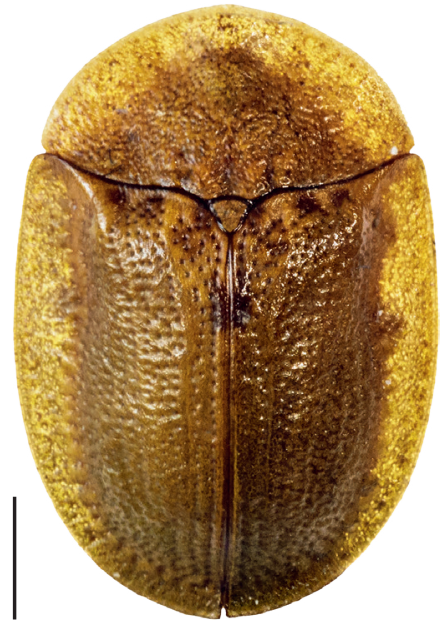

Fig. 5 - Cassida denticollis (Photo I. Ș. Iorgu). Scale bar: $1 \mathrm{~mm}$.

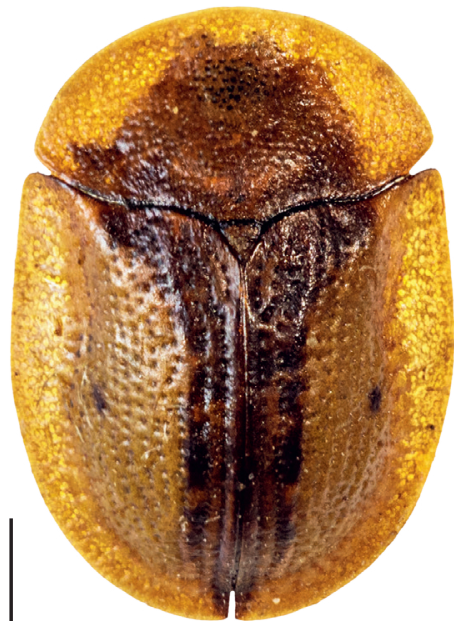

Fig. 6 - Cassida ferruginea (Photo I. Ș. Iorgu). Scale bar: $1 \mathrm{~mm}$. 
Host plants: Asteraceae: Inula britannica, I. dysenterica, I. helenium, I. salicina, Carpesium abrotanoides, C. glossophyllum, Pulicaria prostrata (Borowiec, 2013).

\section{Cassida nebulosa Linnaeus, 1758}

Material: 5 specs, Banat, 4.IV.1884-26.III.1885, without other data; 2 specs, Transylvania, without other data, coll. D.K.; 2 specs, Bucureşti, without other data, coll. R.C.; 3 specs, Bârlad Valley (VS), without other data, coll. A.L.M.; 1 spec., Tuzla (CT), without other data, coll. A.L.M.; 1 spec., Ardeluţa, Tarcău (NT), 24.V.1949, coll. I.C.; 1 spec., Ilişeşti (SV), 20.V.1950, coll. I.C.; 1 spec., Jegălia (CL), 4.IV.1957, coll. I.C.; 1 spec., Valu lui Traian (CT), 1957, X.S.P.; 1 spec., Comorova Forest, Mangalia (CT), 17.VI.1962, N.S.; 1 spec., Valley of the Nera River (CS), 10.VII.1976, A.P.G.; 2 specs, Budeşti (CL), 25.V.1979, G.A.; 1 spec., Pleniţa Forest, Castrele Traiane (DJ), 8.X.1981, G.A.; 4 specs, Valley of the Motru River, Gura Motrului (MH), 21.VI.-19. VII.1983, C.P.; 1 spec., Drobeta-Turnu Severin (MH), 5.VI.1984, R.S.; 6 specs, Carei (SM), 14.V.1985, N.G.; 1 spec., Cărăşeu (SM), 30.VII.1985, V.G.; 3 specs, Băneasa Forest (IF), 12.IV.1988, V.B.; 1 spec., Vasilaţi (CL), 18.V.1988, R.S.; 1 spec., Nuci (CL), V.1988, I.M.; 1 spec., Valea Oglănicului (MH), 300 m a.s.l., 2.VII.1990, Ad.R.; 1 spec., Lupşa Valley, Motru Sec (GJ), 18.VI.1992, N.R.; 1 spec., Caraorman Forest, Danube Delta (TL), 24.VIII.1992, A.P.; 1 spec., Hagieni Forest (CT), 10.VII.1992, C.P.; $1 \mathrm{spec}$,, Negureni Forest (CT), 22.VII.1992, M.A.; Canaraua Fetii (CT), 19-20.V.1993, C.H. (9 specs), 23.VII.1993, G.A. (3 specs), 20.V.1994, G.A. (3 specs); 8 specs, Trăisteni (PH), 22-28.VI.1994, C.P., A.P.; 2 specs, Cârţişoara, Vama Cucului (SB), 2.VIII.1994, C.P.; 3 specs, Călugăreni (GR), 4.V.1996, C.P.; 2 specs, Sinaia, Cumpătu (PH), 27.V.1994, coll. I.C.; 1 spec., "Sfânta Ana" hermitage, Sinaia (PH), VI.1994, coll. I.C.; 1 spec., Pârâul Rece (BV), 9.VII.2004, V.U.

Host plants: Amaranthaceae: Amaranthus ascendens, A. angostanus, A. retroflexus; Chenopodiaceae: Atriplex hastata, A. nitens, A. patula, A. hastatum, $A$. subcordata, Beta vulgaris var. altissima, B. vulgaris var. flavescens, Chenopodium

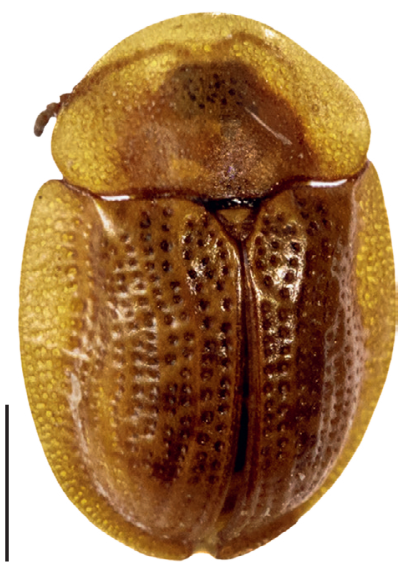

Fig. 7 - Cassida flaveola (Photo I. Ș. Iorgu). Scale bar: $1 \mathrm{~mm}$.

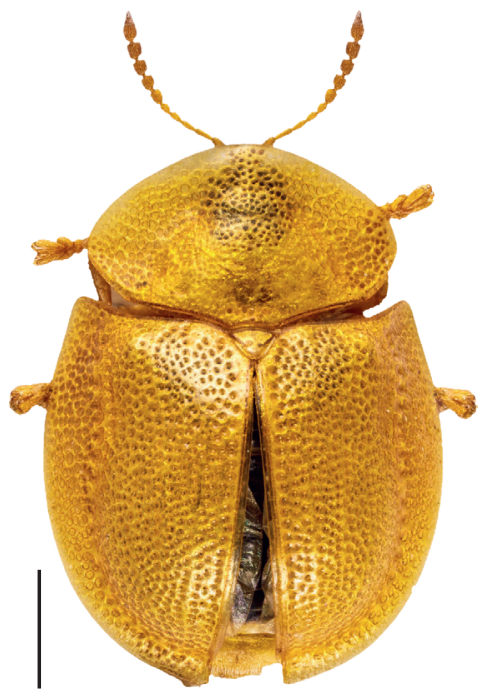

Fig. 8 - Cassida hemispherica (Photo I. Ș. Iorgu). Scale bar: $1 \mathrm{~mm}$. 


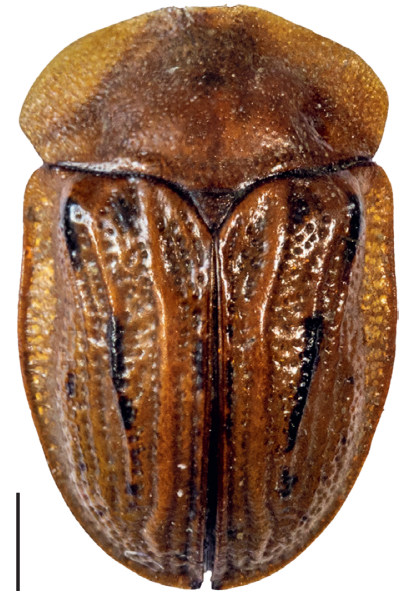

Fig. 9 - Cassida lineola (Photo I. Ș. Iorgu). Scale bar: $1 \mathrm{~mm}$.

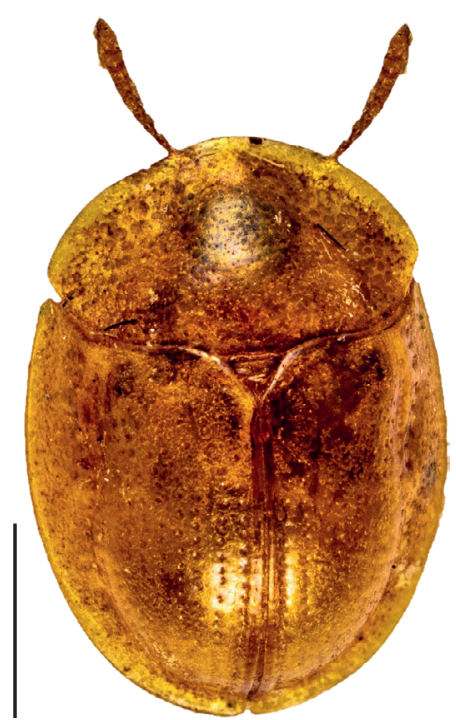

Fig. 10 - Cassida margaritacea (Photo I. Ș. Iorgu). Scale bar: $1 \mathrm{~mm}$.

album, C. album var. centrorubrum, C. rubrum, C. glaucum, C. bonus henricus, C. urbicum, C. polyspermum, C. vulvaria (Borowiec, 2013).

Cassida nobilis Linnaeus, 1758

Material: 1 spec., Herculane Baths (Băile Herculane) (CS), 10.VI.1951, N.S.; București, 20.IV.1955 (1 spec.), 8.V.1983, C.C. (1 spec.), 10.IV.1994, C.H. (1 spec.); 1 spec., Lacul Sărat (BR), 28.V.1955, N.S.; 1 spec., Periprava (TL), 26.VII.1957, I.S.; 2 specs, Şcheia Forest (SV), 11.V.1959, I.N.; 1 spec., Prundu (GR), 2.IX.1976, M.A.; 1 spec., Pasărea Forest, Brăneşti (IF), 2.V.1977, M.A.; 1 spec., Goleşti (AG), 15.IX.1979, I.A.; 1 spec., Purani (TR), 28.IX.1979, G.A.; 1 spec., Tinosu (PH), 14.IV.1981, V.B.; 2 specs, Videle (TR), 11.VII.1986, I.M.; 1 spec., Pasărea Lake (IF), 2.V.2006, C.P.; 1 spec., Caracal (OT), without other data.

Host plants: Asteraceae: Matricaria perforata; Chenopodiaceae: Beta vulgaris, Chenopodium album, C. rubrum; Caryophyllaceae: Silene vulgaris, Spergula arvensis, Stellaria media (Borowiec, 2013).

\section{*Cassida pannonica Suffrian, 1844}

Material: 1 spec., Braşov Mts (Kronstädter Gerbirge), without other data, coll. Fr.D.; 1 spec., Hagieni Forest (CT), 23.VII.1964, M.W. (Fig. 12).

Host plants: Asteraceae: Centaurea jacea, C. paniculata, Cynara scolymus, Erodium sp., Jurinea laxa (Borowiec, 2013).

\section{*Cassida panzeri Weise, 1907}

Material: 1 spec., Piteşti (AG), 3.VI.1960, N.S.; 1 spec., Letea Forest (TL), 9.V.1963, I.S.; 1 spec., Zboina, Vrancei Mts (VN), 10.VI.1971, A.G. (Fig. 13).

Host plants: Asteraceae: Arctium lappa, Cirsium vulgare, C. arvense, Scorzonera humilis, Taraxacum officinale, Tragopogon pratensis (Borowiec, 2013). 
Cassida prasina Illiger, 1798

Material: 1 spec., Postăvaru Mt. (Schuler Gebirge), Braşov (BV), without other data, coll. Fr.D.; 1 spec., Cernica Forest, Bucureşti, 28.V.1955, I.D.; Comana (GR), 30.V.1962, I.S. (1 spec.), 12.VII.1963, Șt.N. (1 spec.); 1 spec., Agigea (CT), 27.V.1963, X.S.P.; 1 spec., Herculane (CS), 24.-27.V.1965, A.P.G.; Pasărea Forest, Brăneşti (IF), 16.VI.1966, A.R. (2 specs), 3.VI.2001, C.P. (1 spec.); 3 specs, Eşelniţa (MH), 17.VI.1970, A.R.; 1 spec., Nucet (DB), 15.VI.1970, I.A.; 1 spec., Perchiu (BC), 12.VI.1971; Cocoş Monastery (TL), 9.V.1973, A.P.G. (1 spec.), 10.VI.1973, A.C. (1 spec.); 3 specs, Hinova (MH), 7.VI.1984, R.S.; 1 spec., Călineşti Oaş (SM), 21.V.1987, R.S.; 1 spec., Tuzla (CT), 1.VIII.1992, A.S.; 1 spec., Dumbrăveni Forest (CT), 18.V.1994, C.H.; 1 spec., 2 specs, Floreşti (GR), 11.VI.1995, C.P.; Celic Dere Monastery (TL), 20.V.1997, C.P., C.H. (13 specs), 27.V.1997, R.S. (1 spec.); 1 spec., Valea Fagilor (TL), 23.V.1997, C.H.; 1 spec., Izvoarele (TL), 21.V.1997, C.P.; 1 spec., Ciucurova Forest (TL), 22.VI.1997, C.H.; 10 specs, Valley of Frumuşeaua River, 7 km upstream Crasna Vişeului (MM), 25.VI.1997, A.S.; 2 specs, Bistra (MM), 25.VI.1997, A,S.; 1 spec., Elma clearing, Repedea (MM), 29.VI.1997, A.S.; 2 specs, Izvorul Dulce, Beceni (BZ), 9.VI.2002, V.U.; 1 spec., Cerna, Măcin Mountains National Park (TL), 26.V.2005, D.R.; 3 specs, Suluc Valley, Măcin Mountains National Park (TL), 27.V.2005, I.M.; 1 spec., Ogarca Forest, Vlaşin (GR), 22.VI.2006, R.S.; 2 specs, Măcin (TL), 10.V.2011, I.Şt.I.; 5 specs, Bârnova Forest (IS), 26.V.2011, I.Şt.I. (Fig. 14).

Host plants: Achillea millefolium, A. ptarmica, Matricaria indora, Tanacetum vulgare (Borowiec, 2013).

\section{Cassida rubiginosa rubiginosa $\mathrm{O}$. F. Müller, 1776}

Material: 1 spec., Transylvania, without other data, coll. D.K.; 1 spec., Haţeg (Hatzeg) (HD), without other data, coll. D.K.; 2 specs, Bucureşti, without other data, coll. R.C.; 2 specs, Tuzla (CT), without other data, coll. A.L.M.; 1 spec., Herculane Baths (Băile Herculane) (CS), 10.VI.1951, N.S.; Câmpulung Moldovenesc (SV), 20.VI.1951, coll. I.C. (3 specs), 4.VI.1952, coll. I.C. (1 spec.); 2 specs, Piteşti (AG), 20.IV.1954, N.S.; Budeşti (CL), 30.V.1954, X.S.P. (3 specs), 25.V.1979, M.W. (1 spec.),

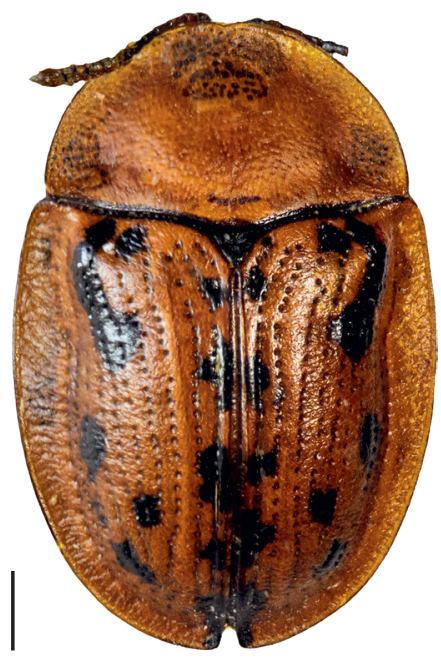

Fig. 11 - Cassida murraea murraea (Photo I. Ș. Iorgu). Scale bar: $1 \mathrm{~mm}$.

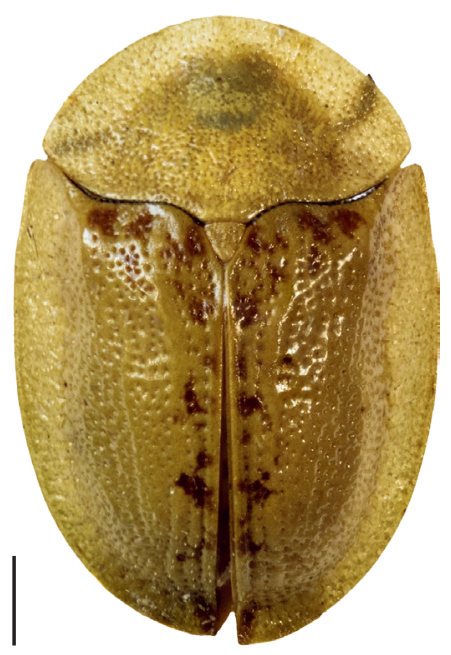

Fig. 12 - Cassida pannonica (Photo I. Ș. Iorgu). Scale bar: $1 \mathrm{~mm}$. 
5-6.VI.1979, M.W., I.D. (5 specs); Căldăruşani (IF), 17.X.1954, X.S.P. (1 spec.), 10.VI.1978, R.S. (1 spec.); Comana Forest (GR), 8.V.1955, N.S. (2 specs), 30.V.1974, A.P.G. (1 spec.), 31.V.1977, G.A. (2 specs), 11.IX.1979, G.A. (1 spec.), 4.VI.1980, C.P. (1 spec.), 19.V.2009, R.S. (2 specs); 1 spec., Simleu Silvaniei (SJ), 12.VIII.1957, X.S.P.; Mogoşoaia Forest (IF), 9.V.1959, V.I.R. (1 spec.), 15.VI.1962, A.R. (1 spec.); Pasărea Forest (IF), 24.V.1962, I.D. (1 spec.), 20.VI.1978, R.S. (1 spec.), 2.V.2006, C.P. (2 specs); 4 specs, Tulcea (TL), 22.VI.1963, X.S.P.; 1 spec., Periprava, Danube Delta (TL), 2.VII.1963, X.S.P.; 1 spec., Chilia Veche (TL), 7.V.1964, I.S.; 1 spec., Agigea (CT), 12.VI.1964, N.S.; 1 spec., Borsec (HD), 11.VI.1967, V.I.R.; 2 specs, Valea Lungă, Câmpina (PH), 27.VII.1967, A.R.; 1 spec., Poiana Stânii, Sinaia (PH), 14.VIII.1967, A.R.; 2 specs, Orşova (CS), 18.VI.1970, A.R.; 1 spec., Ogradena (MH), 21.VI.1970, X.S.P.; 1 spec., Başta (NT), 17.V.1971; 1 spec, Bârlad (VS), 13.V.1973, A.R.; 1 spec., Cocoş Monastery (TL), 16.V.1975, A.C.; 7 specs, Lunca (TR), 31.V.1977, M.A.; 1 spec., Bălăria Forest (GR), 20.VI.1978, R.S.; Zăvestreni Forest (TR), 20.VI.1978, R.S. (2 specs), 3.V.1980, R.S. (2 specs); 1 spec., Râioasa Forest, Chitila (IF), 8.V.1979, G.A.; 4 specs, Căscioarele Forest, Dealu (IF), 7-8.VI.1979, R.S., G.A.; 1 spec., Valley of Argeş River, Buda Cornetu (IF), 2.VIII.1979, M.W.; 1 spec., Bălţata Forest, Miroşi (AG), 15.IV.1980, D.D.; 14 specs, Nanov Forest (TR), 13-14.VI.1980, I.M., R.S., I.D.; 2 specs, Palanca Forest, Nicolae Titulescu (OT), 24.VI.1980, R.S.; 1 spec., Bistreț (DJ), 24.VI.1981, R.S.; 1 spec., Săbăreni (GR), 7.IV.1982; 2 specs, Mușa Forest, DrobetaTurnu Severin (MH), 5.VI.1984, I.M.; 1 spec., Livada (SM), 12.VI.1985, R.S.; 1 spec., Halmeu Vii (SM), 13.VI.1985, R.S.; 2 specs, Căuaş (SM), 29.VI.1985, M A.; 1 spec., Moftin (SM), 1.VII.1985, G.A.; 1 spec., Sâmbăta de Sus (SB), 4.VII.1985, N.G.; 1 spec., Cămârzana (SM), 19.V.1987, C.P.; 3 specs, Dridu (IL), 11.VI.1987, R.S., C.P.; 1 spec., Gălbinaşi (CL), 11.V.1988, R.S.; 1 spec., Canaraua Fetii (CT), 19.V.1993, M.B.; 2 specs, Trăisteni (PH), 23.VI.1994, C.P.; 1 spec., Strâmtura (MM), 3.VII.1995, C.P.; 2 specs, Revărsarea (TL), 23.V.1997, C.P.; 4 specs, Repedea f.c. (MM), 22-26.VI.1997, R.S., C.P.; 2 specs, Crasna Vişeului (MM), 25.VI.1997, R.S.; 2 specs, Galeş (AG),

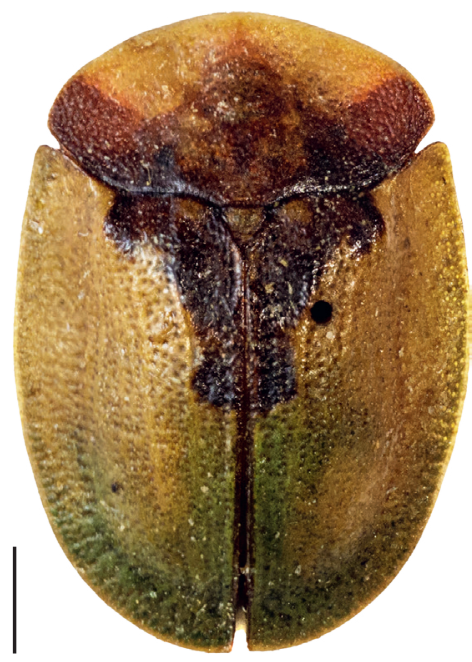

Fig. 13 - Cassida panzeri (Photo I. Ș. Iorgu). Scale bar: $1 \mathrm{~mm}$.

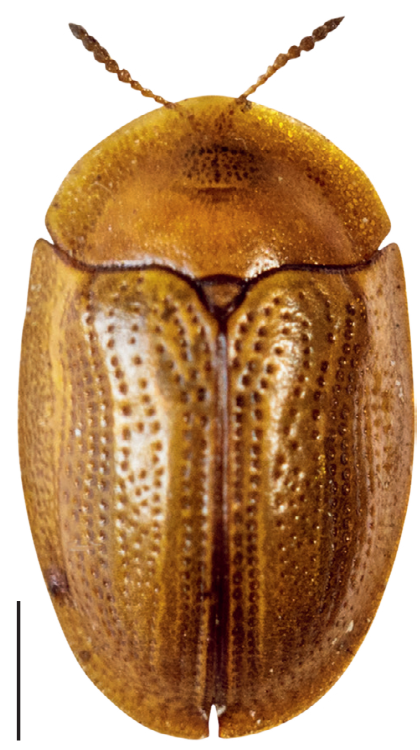

Fig. 14 - Cassida prasina (Photo I. Ș. Iorgu). Scale bar: $1 \mathrm{~mm}$. 
21.VII.2001, C.P.; 2 specs, Valley of Bârsa Mare River, "Piatra Craiului” National Park (BV), 4.VII.2000-2.VII.2002, A.S., C.P.; 1 spec., the confluence of the Rica and Budescu rivers, Poienile de sub Munte (MM), 14.VI.2003, C.P.; 1 spec., Valea Fagilor (TL), 24.V.2005, R.S.; 1 spec., Şaua Sehleanu, the Maramureş Mountains Natural Park (MM), 13.VI.2007, C.P.; 1 spec., Sărata (SB), 4.V.2007, C.B.; 3 specs, Bârnova Forest (IS), 26.V.2011, I.Şt.I.

Host plants: Asteraceae: Arctium lappa, Artemisia maritima, Carduus acanthoides, $C$. argemone ssp. obtusisquamosus, C. crispus, C. nutans, Centaurea jacea, C. pectinata, Cirsium acaule, C. arvense, C. chrysacanthum, C. eryophorum, C. incanum, C. lanceolatum, C. palustre, C. oleraceum, C. rivulare, C. ucrainicus, C. vulgare, Cynara cardunculus, C. scolymus, Inula helenium, Onopordum sp., Pulicaria sp., Saussurea tanakae, Serratula tinctoria, Silybum marianum, Tanacetum sp. (Borowiec, 2013).

\section{Cassida rufovirens Suffrian, 1844}

Material: 1 spec., Braşov, Tâmpa (Dealul Capelei = Kapellenberg) (BV), without other data, coll. Fr.D.; 1 spec., Predeal (BV), 27.X.1964; 1 spec., Eşelniţa (MH), 16.VI.1970, A.R.; 1 spec., Gherăieşti, Bacău (BC), 18.VIII.1972, A.G.; 1 spec., Babadag Forest (TL), 14.VI.1973, A.G.; 2 specs, Piatra Craiului National Park, Sătic (AG), 22.VI.2005, M.S. (Fig. 15).

Host plants: Asteraceae: Achillea sp., Anthemis nobilis, Chamomilla recutita, Matricaria chamonilla, M. indora, M. perforata, M. tzvelevii (Borowiec, 2013).

\section{Cassida sanguinolenta O. F. Müller, 1776}

Material: 1 spec., Siseşti (MH), VI.1954; 3 specs, Greci (TL), 26.V.1961, X.S.P.; 1 spec., Herculane Baths (Băile Herculane) (CS), 14.V.1964, A.P.G.; 1 spec., Pasărea Forest (IF), 16.VI.1966, A.R.; 1 spec., Nucet (DB), 15.VII.1970, I.A.; 1 spec., Valea Crişului Pietros, Bihor Mts. (BH), 1.VII.1976, G.A.; 1 spec., Dumbrăveni Forest (CT), 18.V.1994, C.H.; 5 specs, Valley of Frumuşeaua River, 7 km upstream Crasna Vișeului, 23.V1.1997, R.S.

Host plants: Asteraceae: Achillea millefolium, Tanacetum vulgare (Borowiec, 2013).

*Cassida sanguinosa Suffrian, 1844

Material: 1 spec., Germany, without other data, coll. Ed.R. (Fig. 16).

Host plants: Asteraceae: Achillea ptarmica, A. millefolium, Tanacetum vulgare (Borowiec, 2013).

*Cassida seladonia Gyllenhal, 1827

Material: 1 spec., Chilia Veche (TL), 7.V.1964, I.S. (Fig. 17).

Host plants: Asteraceae: Artemisia arenaria, A. campestris, Filago arvensis, F. gallica, Helichrysum arenarium, H. corymbiformis (Borowiec, 2013).

Cassida stigmatica Suffrian, 1844

Material: 1 spec., without collecting data; 1 spec., Chilia Veche (TL), 7.V.1964, M.W.

Host plants: Asteraceae: Achillea millefolium, Artemisia nitrosa, A. arbotanum, Tanacetum vulgare (Borowiec, 2013). 


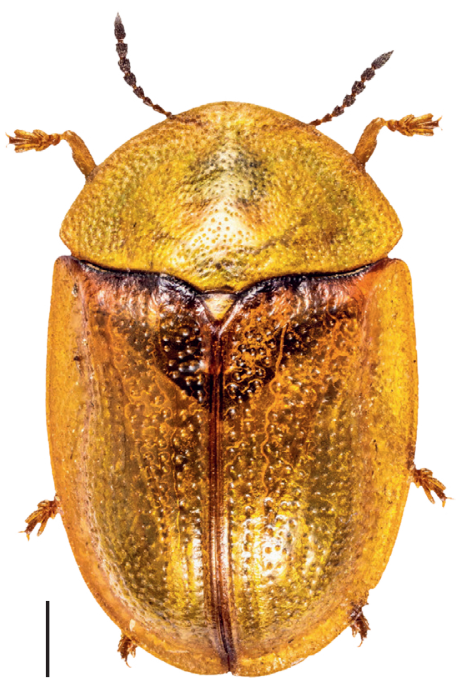

Fig. 15 - Cassida rufovirens (Photo I. Ș. Iorgu). Scale bar: $1 \mathrm{~mm}$

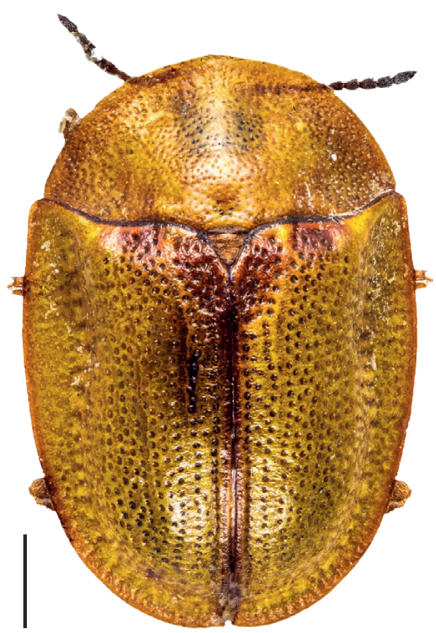

Fig. 16 - Cassida sanguinosa (Photo I. Ș. Iorgu). Scale bar: $1 \mathrm{~mm}$.

*Cassida subreticulata Suffrian, 1844

Material: 1 spec., Vurpăr (Burgberg), 20.V., without other data; 1 spec., Pasărea Forest, Brăneşti (IF), 16.VI.1966, R.A.; 1 spec., Eşelniţa (MH), 20.VI.1970.

Host plants: Caryophyllaceae: Dianthus squarrosus, Melandryum album, Saponaria officinalis, Silene vulgaris, S. dioica (Borowiec, 2013).

Cassida vibex Linnaeus, 1767

Material: 1 spec., Transylvania, without other data, coll. D.K.; 2 specs, Bârlad Valley (VS), without other data, coll. A.L.M.; 1 spec., Budeşti (CL), 3.V.1953, X.S.P.; 3 specs, Ilişeşti (SV), 20.V.1950, coll. I.C.; 1 spec., Esechioi (CT), 19.V.1958, N.S.; 1 spec., Pasărea Forest (IF), 27.V.1958, N.S.; 1 spec., Căldăruşani (IF), 15.VII.1959, I.S.; 1 spec., Periprava, Danube Delta (TL), 11.VII.1960, M.W.; 1 spec., Plesiva, Anina Mts (CS), 24.VI.1962, coll. I.C.; 1 spec., Câmpulung Moldovenesc, Cocou (SV), 20.VII.1962; 1 spec., Tulnici (VN), 22.VII.1962, A.R.; 1 spec., Agigea (CT), 27.V.1963, X.S.P.; 2 specs, Chilia Veche, Danube Delta (TL), 7.V.1964, I.S.; 1 spec., Roşu Mt., Ciucaş Mts. (PH), 7.VII.1966, M.C.; 1 spec., Duruitoarea waterfall, Ceahlău Massif (NT), 9.VIII.1966, M.V.; 1 spec., Dealul Mărului Forest, Roman (NT), 13.VI.1967; 1 spec., Caraorman Forest, Danube Delta (TL), 21.X.1969, M.W.; 1 spec., Eşelniţa (MH), 17.VI.1970, A.R.; 1 spec., Orşova (MH), 18.VI.1970, A.R.; 2 specs, Nanov Forest (TR), 14.VI.1980, I.M.; 1 spec., Strehaia (MH), 11.V.1982, G.A.; 1 spec., Livada (SM), 12.VI.1985, I.M.; 1 spec., Valley of the Bulba River, Baia de Aramă (MH), 17.VI.1982, R.S.; 1 spec., Lupşa Valley, Motru Sec (GJ), 18.VI.1992, N.R.; 1 spec., Canaraua Fetii (CT), 19.V.1993, C.H.; 1 spec., Celic Dere Monastery (TL), 24.V.1997, R.S.; 3 specs, Smereceni clearing, $7 \mathrm{~km}$ towards the Repedea (MM), 22-27.VI.1997, R.S., A.S.; 1 spec., Repedea f.c. (MM), 29.VI.1997, D.R.; 1 spec., Vişeul de Jos (MM), 22.VIII.1997, E.R.; 1 spec., Mara (MM), 21.VII.1998, I.M.; 1 spec., Gârliştea Gorges (CS), 8.VIII.2001, C.P.; 3 specs, Coşnea f.c., Poienile de sub Munte (MM), 15-16. VI.2003, C.P.; 1 spec., Pietrişu island, Danube River at kilometer 760, 16.V.2004, C.P.; 
1 spec., Belciugatele (CL), 13.VII.2005, V.U.; 1 spec., Letea Forest, Danube Delta (TL), 4.V.2012, I.Şt.I.; 2 specs, Sadu (SB), 31.V.2012, I.Şt.I.; 1 spec., Râmeț Gorges, Trascău Mts (AB), 2.VI.2012, I.SSt.I.; 10 specs, Floristic Reserve "The Secular Hayfields from Valea lui David", Iaşi (IS), 10-18.V.2012, I.Şt.I.; 1 spec., Lepşa (VN), 30.V.2015, A.L.; 2 specs, Valea Strâmbă, Lepşa (VN), 31.V.2015, A.L.

Host plants: Asteraceae: Arctium lappa, A. minus, A. nemorosum, Carduus acanthoides, Cirsium arvense, $C$. oleraceum, C. palustre, $C$. rivulare, C. lanceolatum, Centaurea carpathica, C. scabiosa, Tanacetum vulgare (Borowiec, 2013).

\section{Cassida viridis Linnaeus, 1758}

Material: 1 spec., Gorj County, without other data, coll. R.C.; 2 specs, Vâlcea County, without other data, coll. R.C.; 3 specs, Carpathian Mountains (Moldavia), without other data, coll. A.L.M.; Herculane Baths (Băile Herculane) (CS), 1950, coll. E.V. (1 spec.), 15.V.1952, N.S. (1 spec.), 20.V.1964, N.S. (1 spec.), 23.VII.1965, N.S. (1 spec.), 4.VII.1968, V.B. (4 specs), 28.V.1974, coll. I.C. (1 spec.); 3 specs, Cernica Forest (IF), V.1945, coll. I.C.; 1 spec., Câmpulung Moldovenesc, Deia (SV), 27.V.1951, coll. I.C.; 1 spec., Gura Humorului (SV), 15.X.1951, coll. I.C.; 1 spec., Bârnova Forest (IS), 2.VI.1953, N.S.; 1 spec., Chitila (Bucureşti), 19.V.1953, X.S.P.; 1 spec., Zăganu Mt., Ciucaş Mts, VII.1953, coll. I.C.; 1 spec., Şandru, Câmpulung Moldovenesc (SV), 23.V.1953, coll. I.C.; 1 spec., Băneasa Forest (IF), 24.V.1954, A.R.; 1 spec., Siseşti (MH), VI.1954; 1 spec., Mogoşoaia Forest (IF), 9.V.1956; 1 spec., Ţigăneşti (Ciolpani) (IF), 10.VIII.1956, X.S.P.; Comana Forest (GR), 31.V.1956 (2 specs), 18.V.1959, I.S. (5 specs), 23.VI.1976 (3 specs), 9.VIII.1979, R.S. (2 specs), 3.VI.1998, M.S.P (1 spec.), 4.V.-7.VI.2006, R.S. (6 specs); 9 specs, Căldăruşani (IF), 31.V.1957, X.S.P.; 2 specs, Timişu de Sus (BV), 21.VI.-4.VII.1957, coll. I.C.; 6 specs, Sinaia (PH), 12.VI.-9. VII.1958, A.R.; 1 spec., Negrişoara (SV), 13.V.1958, coll. I.C.; 1 spec., Baraolt (CV), 1.VIII.1958, coll. I.C.; 4 specs, Omu rivulet, Broşteni (SV), 30.V.1958, coll. I.C.; 3 specs, Timişoara, Green Forest (Pădurea Verde) (TM), 10.V.1960, coll. I.C.; 1 spec., Voineşti (DB), 18.VI.1961, A.R.; 1 spec., Oradea (BH), 30.V.1962, coll. I.C.; 1 spec., Bucureşti, 12.VII.1963, A.R.; 2 specs, Baia Mare (MM), 7.VI.1963, A.R.; 9 specs, Chilia Veche, Danube Delta (TL), 7.V.1964, I.S.; 1 spec., Vaideeni (VL), 10.V.1964, Io.C.; 6 specs, Şarânga clearing, Sinaia (PH), 28.V.1964, Şt.N.; 1 spec., Caraorman Forest, Danube Delta (TL), 5.V.1968, A.P.G.; 1 spec., Domogled Mt. (CS), 2.VI.1965, A.P.G.; 1 spec., Hagieni Forest (CT), 7.VI.1965, Şt.N.; 1 spec., Borsec (HR), 14.VI.1966, X.S.P.; 5 specs, Periprava, Danube Delta (TL), 17.VII.-14.X.1966, X.S.P.; 1 spec., Nanov (TR), 2.X.1966, X.S.P.; 1 spec., Suzana Monastery, Măneciu (PH), 29.VII.1967, X.S.P.; Caraorman Forest, Danube Delta (TL), 21.IX.1967, M.W. (1 spec.), 5.VI.1968, A.P.G. (1 spec.), 29.VIII.1969, M.W. (1 spec.); Putna Valley (SV), 13.V.-17.VI.1971, coll. I.C. (3 specs), 17.V.1972, coll. I.C. (1 spec.), 17.V.1982, coll. I.C. (1 spec.), 23.V.1983, coll. I.C. (2 specs), 22.V.1984, coll. I.C. (1 spec.); 1 spec., Bălătău Lake, Nemira Mts (BC), 10.V.1971; 2 specs, Slănic Moldova (BC), 7.V.-10.VI.1971, M.I.; 2 specs, Sfântu Gheorghe, Danube Delta (TL), 9.VIII.1972, A.P.G.; 1 spec., Gâdinţi (NT), 20.VII.1972; 1 spec., Başta (NT), 23.V.1973; 2 specs, Pasărea Forest (IF), 30.VI.1978, G.A.; 1 spec., Drăgăneşti Vlaşca (TR), 20.VI.1979, M.W.; 1 spec., Căldăraru Forest (TR), 27.IX.1979, R.S.; 1 spec., Purani (TR), 27.IX.1979, G.A.; 1 spec., Gura Motrului (MH), 29.VII.1981, I.M.; 1 spec., Cobia Forest (DJ), 19.VI.1981, C.P.; 1 spec., Poiana Mare (DJ), 6.VIII.1982, C.P.; 1 spec., Perişor (DJ), 24.IX.1982, G.A.; 2 specs, Vânju Mare (MH), 15.VII.1983, I.M.; 1 spec., Hinova (MH), 7.VI.1984, I.M.; 1 spec., Cărăşeu (SM), 30.VII.1985, V.G.; Poiana Neamţului (SB), 19-20.VII.1985, I.M., R.S. (6 specs), 
14-15.V.1986, I.M., I.D. (14 specs); 5 specs, Turnu Roşu (SB), 13.V.1986, I.M.; 2 specs, Călineşti Oaş (SM), 21.V.1987, R.S.; 2 specs, Vatra Dornei (SV), 30.V.1989, V.B.; 1 spec., Valea Drăganului (CJ), 8.VIII.1989, R.S.; 2 specs, Giuleşti Sârbi (IF), 8.VII.1991; 2 specs, Cloşani (GJ), 16.VI.1992, C.P.; 1 spec., Doloşman Cape, Razim (Razelm) Lake (TL), 11.V.1992, N.G.; Hagieni Forest (CT), 22.V.1993, C.P. (1 spec.), 18.VII.1997, C.P. (2 specs); 1 spec., Aleșd, Plopiş Mts (BH), 24.IV.1994, A.S.; 2 specs, Trăisteni (PH), 22-28.VI.1994, A.P.; 1 spec., Vama Cucului, Făgăraş Mts, 1.VIII.1994, C.H.; 1 spec., Strâmtura (MM), 3.VII.1995, C.P.; 2 specs, Călugăreni (GR), 4.V.1996, C.P.; 2 specs, Smereceni clearing, $7 \mathrm{~km}$ towards the Repedea (MM), 22.VI.1997, R.S., A.S.; 5 specs, Repedea f.c. (MM), 22.VI.-2.VII,1997, R.S., A.S., I.M.; 7 specs, Elma clearing, Repedea (MM), 24-29.VI.1997, R.S., A.S., C. P.; 1 spec., Valea Teilor (TL), 22.V.1997, C.P.; 1 spec., Revărsarea (TL), 23.V.1997, C.P.; 9 specs, Valley of Frumuşeaua River, 7 km upstream Crasna Vişeului, 23.V1.1997, C.P., R.S.; 1 spec., Buzău (BZ), 17.IV.2000, V.U.; 1 spec., Bârsa Groşetului, 4 km upstream Plaiul Foii, Piatra Craiului National Park (BV), 9.VI.2000, C.P.; Valley of Bârsa River, Piatra Craiului National Park (BV), 4.VII.2000, L.O.P. (2 specs), 24.V.2004, R.S. (2 specs); 1 spec., Piscul cu Brazi, Piatra Craiului National Park (BV), 5.VII.2000, L.O.P.; 1 spec., Bârsa Fierului Valley, Piatra Craiului National Park, 24.V.2004, C.B.; 3 specs, the confluence of the Rica and Budescu rivers, Poienile de sub Munte (MM), 14.VI.2003, C.P.; 2 specs, 17 km far from Reşiţa, towards Văliug (CS), 10.VIII.2001, A.S.; $1 \mathrm{spec}$., Valley of Budescu Mare River, Poienile de sub Munte (MM), 14.VI.2003, M.S.; 1 spec., Coşnea f.c., Poienile de sub Munte (MM), 16.VI.2003, C.P.; 2 specs Valley of the Pop Ivan River, $11 \mathrm{~km}$ upstream Crasna Vişeului (MM), 17.VI.2003, R.S., D.S.; 1 spec., Crasna Vişeului (MM), 17.VI.2003, M.S.; 2 specs, Repedea f.c. Repedea (MM), 18.VI.2003, R.S., C.P.; Făina, Vaser Valley Forestry Railway (MM), 19.VI.2003, R.S., C.P. (2 specs), 21.VII.2004, C.P. (1 spec.); 3 specs, Baicu f.c., Ţibleş Mts, Dragomireşti (MM), 22.VI.2003, R.S.; 1 spec., Pălcuţ rivulet, Dragomireşti (MM), 22.VI.2003, C.P.; 3 specs, Țibleş Mts, Dragomireşti, Lunca lui Cosiţă (MM), 22.06.2003, R.S.; 3 specs, Zărneşti Gorges, Piatra Craiului National Park (BV), 22.V.2004, C.B., C.P.; 1 spec., Piatra Craiului National Park, Sătic, Onceoaia Valley (AG), 23.V.2004, S.R.; 1 spec., Făgăraş Mts, Otic Valley, 4.VIII2004, R.S.; 2 specs, Piatra Craiului National Park, Brusturet chalet, Valea Seacă (AG), 21.VI.2005, C.B.; 1 spec., Piatra Craiului National Park, Sătic (AG), 22.06.2005, M.S.; 1 spec., Piatra Craiului National Park, Valea cu Apă, 23.VI.2005, E.I.; 4 specs, Preluca (MM), 12.VI.2007, C.P.; 1 spec., Letea Forest, Danube Delta (TL), 22.IX.2010, I.Şt.I.; 1 spec., Rodina (CS), 20.VII.2011, I.Şt.I.; 2 specs, Jidoaia (VL), 21.V.2014, E.I.; 1 spec., Lepşa (VN), 30.V.2015, A.M.K.

Host plants: Lamiaceae: Galeopsis grandiflora, G. speciosa, G. tetrahit, G. pubescens, Isodon kameba, Lycopus europaeus, Melissa officinalis, Mentha aquatica, $M$. arvensis, $M$. longifolia, $M$. rotundifolia, $M$. suaveolens, $M$. verticillata, Nepeta cataria, Salvia glutinosa, S. officinalis, S. pratensis, S. palustris, S. recta, S. silvatica (Borowiec, 2013).

Cassida vittata Villers, 1789

Material: 1 spec., whitout collecting data, coll. I.C.

In Romania, this species was recorded from Rodnei Mts (Petri, 1912); Sibiu and Reghin (Kuthy, 1897); Bucharest (Serafim \& Maican, 2008).

Host plants: Asteraceae: Anthemis arvensis, Matricaria perforata; Chenopodiaceae: Atriplex halimus, A. prostrata, Chenopodium album, C. rubrum, 
Salicornia sp., Salsola sp., Spinacia oleracea; Urticaceae: Urtica dioica; Caryophyllaceae: Arenaria maritima, Spergula arvensis, Spergularia salina (Borowiec, 2013).

\section{Pilemostoma Desbrochers Des Loges, 1891 \\ *Pilemostoma fastuosum (Schaller, 1783)}

Material: 1 spec., Transylvania, without other data, coll. D.K.; 1 spec., Greci (TL), 22.VI.1963, I.S.; 1 spec., Letea Forest, Danube Delta (TL), 12.V.1964, X.S.P.; 1 spec., Herculane Baths (Băile Herculane) (CS), 19.V.1964, A.P.G.; 2 specs, Gâdinți (NT), 23.V.1965; 1 spec., Caraorman Forest, Danube Delta (TL), 5.V.1968, A.P.G.; 1 spec., Başta (NT), 23.V.1973; 3 specs, Floristic Reserve "The Secular Hayfields from Valea lui David", Iași (IS), 9-10.VI.1984, I.D., A.P.G. (Fig. 18).

Host plants: Asteraceae: Inula sp., Pulicaria dysenterica, P. vulgaris, Senecio jacobaea (Borowiec, 2013).

Tribe Hispini Gyllenhal, 1813

Dicladispa Gestro, 1897

Dicladispa testacea (Linnaeus, 1767)

Material: 1 spec., Turkey, Kahmaranmaraş Province, Kahmaranmaraş, 570 m a.s.1., 1.VI.2008, C.P. (“Euphrates" Expedition).

Host plants: Cistaceae: Cistus sp., Halimium sp., Helianthemum sp.; Anacardiaceae: Pistacia lentiscus (Biondi et al., 1995; Świętojańska et al., 2014).

Hispa Linnaeus, 1767

Hispa atra Linnaeus, 1767

Material: 1 spec., without collecting data, coll. I.C.; 1 spec., Mireşu Mare (Nagynyires) (MM), without other data, coll. E.V.; 3 specs, Cetăţuia, Danube River at kilometer 516 (GR), 26.IV.2004, M.S.; 1 spec., Bucureşti, Cişmigiu Park, IX.2007, S.St.

Host plants: Poaceae: Poa sp., Agropyron sp. (Mohr, 1966).

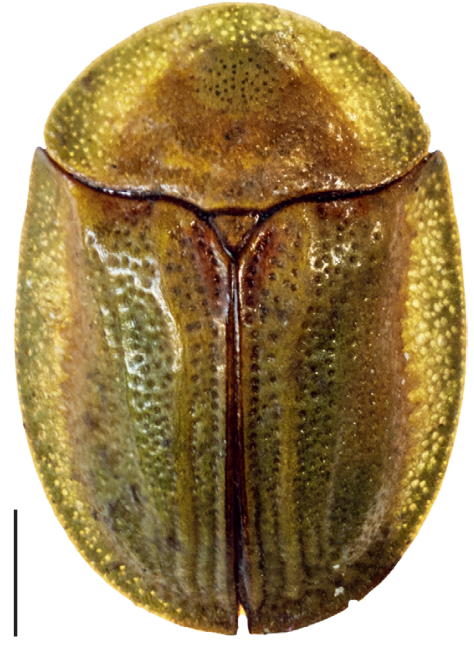

Fig. 17 - Cassida seladonia (Photo I. Ș. Iorgu). Scale bar: $1 \mathrm{~mm}$.

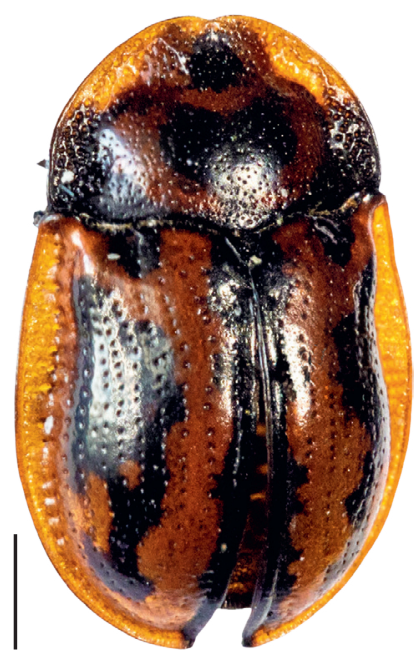

Fig. 18 -Pilemostoma fastuosum (Photo I. Ș. Iorgu). Scale bar: $1 \mathrm{~mm}$ 
The new Chrysomelidae collection of the "Grigore Antipa" National Museum of Natural History preserves 31 species of Cassidinae belonging to genera Cassida (28 species), Pilemostoma, Hispa and Dicladispa (each with one species), representing over $90 \%$ of the total number of species recorded in the Romanian fauna. Out of them, 11 species recently entered in the Museum collections.

A number of 20 species are also found in the Collection of Palaearctic Coleoptera, represented mainly by the material collected by Eduard Fleck and Arnold Lucien Montandon. The Eduard Fleck collection (acquired by the museum in 1914) includes one specimen of Ischyronota desertorum Gebler, labeled "Azuga", without other collecting data. Unfortunately, in the collection many labels are printed with "Azuga", however this does not mean that the specimens were collected from this locality.

Common species of Romania (e.g., Cassida murraea Linnaeus, C. rubiginosa Müller, C. vibex Linnaeus, $C$. viridis Linnaeus, C. nebulosa Linnaeus) are represented in the collection through a great number of specimens.

Some rare species in the Romanian fauna are newly entered in the collections of "Grigore Antipa" Museum, such as: Cassida atrata Fabricius, C. azurea Fabricius, $C$. berolinensis Suffrian, C. margaritacea Schaller, C. pannonica Suffrian, C. seladonia Gyllenhal, C. subreticulata Suffrian.

Most of the material was collected from Romania. The collection also includes some specimens from Slovenia (of the species Cassida aurora Weise), Czechia (Cassida denticollis Suffrian and C. ferruginea Goeze), Germany (C. sanguinosa Suffrian), Tunisia (Cassida deflorata Suffrian) and Turkey (Cassida murraea Linnaeus and Dicladispa testacea Linnaeus).

Cassida deflorata Suffrian, represented in the collection by one specimen from Tunisia, is a Mediterranean species, distributed in north-western Africa, Iberian Peninsula, Sardinia, Sicily and Italy (Warchałowski, 2010). Fleck (1905), most probably erroneously, mentioned it from Romania (Bucharest and Băneasa). The records of this species in Romanian fauna is questionable.

Dicladispa testacea Linnaeus, a species distributed in the Mediterranean area, is represented in the collection by the material collected from Turkey, in the "Euphrates" Expedition.

\section{ACKNOWLEDGMENTS}

The authors express sincere thanks to Dr. Melanya Stan ("Grigore Antipa" National Museum of Natural History, Bucharest) for granting access to the collections, and to Dr. Ottó Merk1 (Hungarian Natural History Museum, Budapest) for his constructive comments on the manuscript. Many thanks to Dr. Ionuţ Ştefan Iorgu and Dr. Tiberiu Sahlean for photographies, and also to Rada Tiţă, Marinela Năzăreanu, Marcela Bugnar, Mariana Foaltin and Dana Ciopec for their help in preparation and labeling of the specimens and the proper conservation of collections. A part of this study was funded by project no. RO1567-IBB01/2017 from the Institute of Biology Bucharest of Romanian Academy.

\section{REFERENCES}

BIONDI, M., R. REGALIN, M. DACCORDI, R. POGGI (1995) I Crisomelidi (esclusi Alticini) delle Isole Circumsarde. Annali del Museo Civico di Storia Naturale "G. Doria", 90: 629-651. (in Italian) BOROWIEC, L. (1999) A world catalogue of the Cassidinae (Coleoptera: Chrysomelidae). Biologica Silesiae. Wroclaw, 476 pp.

BOROWIEC, L. (2013) Website resource of the Department of Biodiversity and Evolutionary Taxonomy, University of Wroclaw. Available at: http://culex.biol.uni.wroc.pl/cassidae/European\% 20Chrysomelidae/index.htm. Downloaded on 5 August 2017. 
BOROWIEC, L., L. SEKERKA (2010) Subfamily Cassidinae Gyllenhal, 1813. Pp. 368-389. In: I. Löbl, A. Smetana (eds), Catalogue of Palaearctic Coleoptera, Vol. 6, Chrysomeloidea. Apollo Books, Stenstrup, $924 \mathrm{pp}$.

BOROWIEC, L., J. ŚWIĘTOJANSKA (2002-2017) Cassidinae of the world - an interactive manual (Coleoptera: Chrysomelidae). Department of Biodiversity and Evolutionary Taxonomy, University of Wroclaw, Poland. Available at: http://www.biol.uni.wroc.pl/cassidae/ katalog\%20internetowy/index.htm Downloaded on 5 August 2017.

BOROWIEC, L., J. ŚWIĘTOJANSKA (2014). Cassidinae Gyllenhal, 1813. Pp. 198-217. In: R.A.B. Leschen, R.G. Beutel (eds.) Coleoptera Beetles, Vol. 3, Morphology and Systematics: Phytophaga. Walter de Gruyter GmbH \& Co KG, Göttingen, 675 pp.

CHABO, C.S. (2007). Biology and phylogeny of the Cassidinae Gyllenhal sensu lato (tortoise and leaf-mining beetles) (Coleoptera: Chrysomelidae). Bulletin of the American Museum of Natural History, 305: 1-250.

FLECK, E. (1905) Die Coleopteren Rumäniens. Bulletin de la Société des Sciences de Bucarest, 14: 695-729. (in German)

HOINIC, C. (1994) A review of the species of Macroplea Samouelle (Coleoptera: Chrysomelidae) in Romania. Travaux du Muséum d'Histoire Naturelle "Grigore Antipa", 34: 17-30.

KUTHY, D. (1897) Ordo Coleoptera. In: A Magyar Birodalom Állatvilága (Fauna Regni Hungariae). III. Arthropoda. (Insecta. Coleoptera.). Királyi Magyar Természettudományi Társulat, Budapest, 213 pp. (in Hungarian and Latin).

MAICAN, S. (2005) Checklist of Chrysomelidae (Coleoptera) of Romania. Travaux du Muséum National d'Histoire Naturelle "Grigore Antipa", 48: 119-136.

MAICAN, S. (2006) Clytrins (Coleoptera: Chrysomelidae: Clytrinae) from the collections of the "Grigore Antipa" National Museum of Natural History (Bucharest). Travaux du Muséum National d'Histoire Naturelle "Grigore Antipa", 49: 239-257.

MAICAN, S. (2007a) Some mediterranean Chrysomelids species (Coleoptera: Chrysomelidae) newly entered in the collections of "Grigore Antipa" National Museum of Natural History [Results of expeditions from Turkey and Tunisia, 2005-2006]. Travaux du Muséum National d'Histore Naturelle "Grigore Antipa", 50: 421-429.

MAICAN, S. (2007b) Contributions to the knowledge of the leaf beetle fauna (Coleoptera: Chrysomelidae) from Maramureş (northern Romania). Entomologica Romanica, 12: 301-324.

MAICAN, S., R. SERAFIM (2001) Chrysomelidae (Coleoptera) from Maramureş. Travaux du Muséum National d'Histoire Naturelle "Grigore Antipa", 43: 199-233.

MAICAN, S., R. SERAFIM (2004) Leaf-beetles (Coleoptera: Chrysomelidae) from Maramureş (Romania). Travaux du Muséum National d'Histoire Naturelle "Grigore Antipa", 46: 139-159.

MAICAN, S., R. SERAFIM (2009) Data about Cerambycidae and Chrysomelidae (Coleoptera: Chrysomeloidea) collected from mediterranean expeditions [Results of the "Taurus" 2005, "Focida" 2006, "Punia" 2006, "Atlas" 2007 and "Euphrates" 2008 expeditions]. Travaux du Muséum National d'Histoire Naturelle "Grigore Antipa", 52: 415-428.

MAICAN, S., R. SERAFIM (2012) Overview on the Chrysomeloidea superfamily (Coleoptera: Cerambycidae, Orsodacnidae, Chrysomelidae) in Dobrogea (Romania). Travaux du Muséum National d'Histoire Naturelle "Grigore Antipa", 55 (1): 65-123.

MAICAN, S., R. SERAFIM (2016) The catalogue of Donaciinae and Criocerinae species (Coleoptera: Chrysomelidae) from the new leaf beetle collection from "Grigore Antipa" National Museum of Natural History (Bucharest) (Part I). Travaux du Muséum National d'Histoire Naturelle "Grigore Antipa", 59 (2): 179-194.

MOHR, K.-H. (1966) Familie Chrysomelidae. Pp. 95-280. In: H. Freude, K. W. Harde, G. A. Lohse (eds.), Die Käfer Mitteleuropas, Band 9, Cerambycidae, Chrysomelidae. Goecke \& Evers Verlag, Krefeld, 299 pp.

NEGRU, ŞT. (1968) Ord. Coleoptera (pars). In: Entomofaune de l'Ile de Letea (Delta du Danube). Travaux du Muséum d'Histoire Naturelle "Grigore Antipa", 9: 81-95. (in French)

NEGRU, ŞT., A. ROŞCA (1967) Ord. Coleoptera. In: L'entomofaune des forêsts du sud de la Dobroudja. Travaux du Muséum d'Histoire Naturelle "Grigore Antipa", 7: 119-148. (in French)

PETRI, K. (1912) Siebenbürgens Käferfauna auf Grund ihrer Erforschung bis zum Jahre 1911. Verhandlungen und Mitteilungen des Siebenbürgischen Vereins fur Naturwissenschaften zu Hermannstadt: $1-376$.

SEENO, T., J. WILCOX (1982) Leaf-Beetle Genera (Coleoptera: Chrysomelidae). Entomography Publications, Sacramento, California, $221 \mathrm{pp}$. 
SEKERKA, L. (2007) Detailed distribution of Cassida sanguinosa and C. leucanthemi (Coleoptera: Chrysomelidae: Cassidinae: Cassidini). Acta Entomologica Musei Nationalis Pragae, 47: 203-209.

SEKERKA, L. (2010) Icones Insectorum Europae Centralis. Coleoptera: Chrysomelidae: Cassidinae. Folia Heyrovskyana, Serie B, 13: 1-24.

SERAFIM, R., S. MAICAN (2004) Contributions to the knowledge of the Coleopterans from the littoral of the Black Sea (Romania). Travaux du Muséum National d' Histoire Naturelle "Grigore Antipa", 47: 169-210.

SERAFIM, R., S. MAICAN (2008) Data on Cerambycidae and Chrysomelidae (Coleoptera: Chrysomeloidea) from Bucureşti and surroundings. Travaux du Muséum National d'Histoire Naturelle "Grigore Antipa", 51: 387-416.

SERAFIM, R., S. MAICAN (2011) Catalogue of Cerambycidae, Megalopodidae and Chrysomelidae (Coleoptera: Chrysomeloidea) recently entered in the patrimony of "Grigore Antipa" National Museum of Natural History (Bucharest). Igor Ceianu collection. Travaux du Muséum National d'Histoire Naturelle "Grigore Antipa", 54 (2): 425-460.

STAINES, C.L. (2002) The New World tribes and genera of hispines (Coleoptera: Chrysomelidae: Cassidinae). Proceedings of the Entomological Society of Washington, 104 (3): 721-784.

ŚWIĘTOJAŃSKA, J., L. BOROWIEC, M. STACH (2014) Redescription of immatures and bionomy of the Palaearctic species Dicladispa testacea (Linnaeus, 1767) (Coleoptera: Chrysomelidae: Cassidinae: Hispini), a leaf-mining hispine beetle. Zootaxa, 3811 (1): 001-033.

UNGUREANU, V., S. MAICAN, R. SERAFIM (2008) Diversity of coleopterans: Cerambycidae, Chrysomelidae, Coccinellidae from Buzău area (Romania). Travaux du Muséum National d'Histoire Naturelle "Grigore Antipa", 51: 171-183.

WARCHAŁOWSKI, A. (2003) Chrysomelidae. The leaf beetles of Europe and the Mediterranean area. Natura Optima dux. Foundation, Warszawa, $600 \mathrm{pp}$.

WARCHAŁOWSKI, A. (2010) The Palaearctic Chrysomelidae. Identification keys. Volumes 1 \& 2. Natura Optima dux. Foundation, Warszawa, 1212 pp. 